\title{
The verification of a new approach to the experimental estimation of tensile forces in prestressed structural elements by method based on the magnetoelastic principle
}

\author{
Tomáš Klier ${ }^{1}$, Tomáš Míčka ${ }^{1}$, Tomáš Plachý2 ${ }^{2}$, Michal Polák ${ }^{2, *}$, Tomáš Smeták ${ }^{3}$ and Miloš \\ Šimler $^{3}$ \\ ${ }^{1}$ Pontex spol. s r. o., Bezová 1658, 14714 Prague 4, Czech Republic \\ ${ }^{2}$ Czech Technical University in Prague, Faculty of Civil Engineering, Thákurova 7, 16629 Prague 6, \\ Czech Republic \\ ${ }^{3}$ Freyssinet CS, a. s., Zápy 267, 25001 Brandýs nad Labem, Czech Republic
}

\begin{abstract}
There are a large number of civil engineering structures where the important structural elements are loaded by large tensile forces. In many practical cases, it is significant to know the current value of tensile force or prestressed stress in these elements for accurate assessment of the reliability of the whole structure. The brief principle and the basic validation results of the new approach to the experimental estimation of tensile forces or stress in prestressed structural elements of engineering structures by the method based on the magnetoelastic principle are described in the paper. The new approach was designed and developed especially for application on existing prestressed concrete structures.
\end{abstract}

\section{Introduction}

The basic objectives of the solved problems were research, development, verification and validation of the new approach to experimental estimation of tensile forces in prestressed structural elements produced from ferromagnetic materials, as are prestressed strands, wires and bars, on engineering structures by method based on the magnetoelastic principle.

Structural elements loaded by large tensile forces, as are prestressed reinforcements of prestressed concrete bridges, stays of cable-stayed bridges, suspension cables and vertical suspenders of suspension bridges, suspension cables of suspended roofs, guy wires of radio masts, ground and rock anchors for example, are usually important structural members of a civil engineering construction that influence generally significantly the load bearing capacity of the whole structure. The tensile force in these elements varies in time substantially. The knowledge of its current accurate value is important for exact assessment of the reliability of the entire structure in any of its life cycle, as are its building, putting into operation, monitoring of its construction conditions during its service, preparation of its reconstruction, et cetera.

${ }^{*}$ Corresponding author: polak@fssv.cvut.cz 
The basic intention of the research work described in this paper was a solution of the problem that could be entitled "an accurate experimental estimation of the current value of the tensile force in a particular prestressed structural element on an existing prestressed concrete structure". This task is very common in civil engineering praxis especially in prestressed concrete bridges but is not frequently satisfactorily soluble by any other experimental or theoretical method.

\section{Brief description of the magnetoelastic method}

\subsection{The physical principle}

The method is based on an experimental estimation of the magnetic response of the tensile stressed structural element on an external magnetic field. It means, the method is applicable for elements made from ferromagnetic materials only, as is the steel used for production of prestressed reinforcements for example.

The magnetic field intensity $H$ and the magnetic flux density $B$ are ones of the basic physical quantities describing the magnetic field arrangement. The magnetic flux density $B$ compared to the magnetic field intensity $H$ describes a force effect of the magnetism. The hysteresis loop characterizes the relation between $B$ and $H$ which depends on the quantity called the magnetic permeability of material $\mu$ that is influenced by particular materials subjected to the effect of the magnetic field and by immediate state of these materials. Some materials or surroundings amplify the force effect of the magnetic field more or less, some others on the contrary fade it.

As was said above, the permeability is given by the kind of material exposed to the effects of the magnetic field, its properties and current conditions. Generally, the value of permeability depends on the level of the affected magnetic field, and not only on its actual value, but also on its history and rate of change, on the actual material temperature and on the current material stress. The ferromagnetic materials are the typical example of ones with this general dependence.

\subsection{The principle of the method and the new approach}

As was said above, the method described in this paper is based on the magnetoelastic principle. It is known, the magnetic parameters of the ferromagnetic materials are dependent on the level of their mechanical stress among others. The practical consequence is the permeability of steel, what is the function of the tension force or the stress acting on the observed steel structural element and this fact is used by the magnetoelastic method for evaluation of its mechanical stress or tension force.

The principle of the newly proposed arrangement of the magnetoelastic sensor is shown in Fig. 1. The first part of the sensor is the controlled source of the variable homogeneous magnetic field, as is the primary coin supplied by varying current. The second part is the appropriately structured system of Hall sensors for detailed observation of the magnetic field intensity $H$ in the investigated cross section of the studied prestressed structural element. The third one is the secondary coil scanning the magnetic flux density $B$ in the investigated cross section.

The Hall sensor is an electronic component that is used for measuring of the magnetic field intensity $H$. The modern Hall sensors are small in size and they are sufficiently sensitive for accurate enough observation of the tangential component of the magnetic field close to the surface of the studied prestressed structural element. 


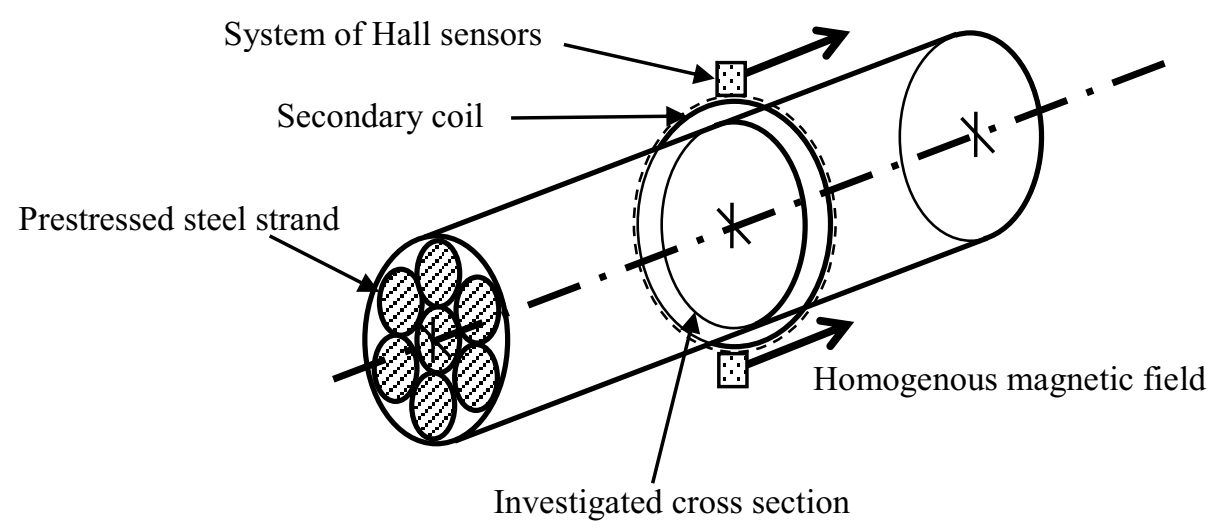

Fig. 1. The principle of the newly proposed arrangement of the magnetoelastic sensor.

\section{The verification of the new approach}

\subsection{Description of the experiments}

A number of laboratory experiments was realized during the verification of the new approach to experimental estimation of tensile forces in prestressed structural elements based on the magnetoelastic principle.

Various possibilities for sensor configuration and measurement parameters were examined.

For example, an appropriate placement of the Hall sensors around the observed element for satisfactory characterization of the magnetic field intensity $H$ in the immediate vicinity of the element, an influence of the magnetic surroundings, an effect of the sensor shielding, a suitable time behaviour and time duration of the varying current signal supplying the primary coil and the most suitable dimensionless parameter $P$ used for results evaluation were investigated.

The verification of the new approach was completed by a laboratory experiment that was focused on the systematic investigation of changes in the magnetic behaviour of selected prestressed elements in dependence on its instant temperature and level of the mechanical stress. The experiment was carried out in November 2016 in the experimental centre of the Klokner institute (the Czech Technical University in Prague). The magnetoelastic characteristics of eight prestressed elements were studied during the experiment. Five elements using at present time (two prestressed strands Lp15,7/1860 MPa (below labelled L1 and L2) and three prestressed bars 15/17 made by firms Dywidag and Mikusol (below labelled T1, T2 and T3)) and three elements that were used formerly and were obtained by demolitions of old structures made from prestressed concrete (three patented wires P4,5 of different age (below labelled D1, D2 and D3)), were investigated.

During the experiment, the studied elements were placed in a climatic chamber (see Fig. 2) and loaded in a steel tensile testing machine (see Fig. 2).

The magnetic behaviour of the investigated elements was observed in four temperature steps namely, at $-20{ }^{\circ} \mathrm{C}$, at $\pm 0{ }^{\circ} \mathrm{C}$, at $+20{ }^{\circ} \mathrm{C}$ and at $+40{ }^{\circ} \mathrm{C}$. In each temperature step, the elements were loaded in nine or seven force steps selected differently for each particular element according to its design resistance.

For each element and each particular temperature and force step, the hysteresis loop was measured and evaluated. With respect to the quantity and complexity of the obtained experimental results in the form of the hysteresis loops, it was not effective to evaluate 
measured data in their whole range. At first, several definitions of the different dimensionless parameters $P$ were defined. From now on, they represented the shape and character of each measured hysteresis loop.

Secondly, the dependence of the different evaluated parameters $\mathrm{P}$ on the temperature and the mechanical stress was statistically evaluated and critically assessed. Base on this rating, the resultant dimensionless parameter $\mathrm{P}$ was chosen that is defined as the difference between two values of the quantity B in exactly determined points of the hysteresis loop divided by the greater quantity B. It has been used for finally evaluation of all measured data.
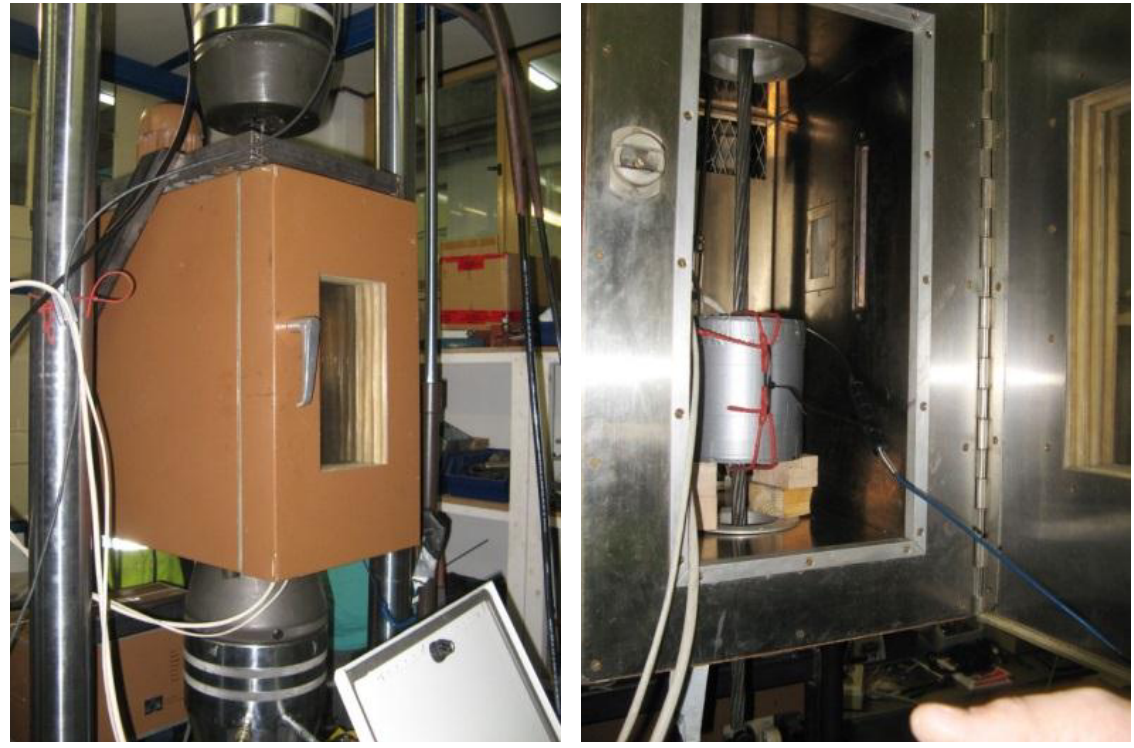

Fig. 2. The exterior view on the climatic chamber and the steel tensile testing machine (on left) and on the magnetoelastic sensor installed on the prestressed strand inside the chamber (on right).

\subsection{The conclusions resulted from the verification experiments}

The surface appearance of the investigated patent wires D1, D2 and D3 does not allow to differ reliably their kind during a real application of the method on an existing structure though the differences in chemical composition were found between the particular wires (see Table 1). For this reason, the results relation between the stress and parameter $P$ (see Fig. 4) was evaluated as the average value for all three studied wires. However, as expected, the evaluated magnetoelastic characteristics for the particular wires have differed slightly each other. But their differences were small and the values of the stress evaluated based on the average relation between the stress and parameter $P$ deviated from the real values about $\pm 3 \%$ of the largest stress at maximum.

During the verification experiments, the magnetoelastic characteristics of the prestressed strands were studied for one kind of this material only which is used in the civil engineering practice currently. However, several different test samples of this material were used. The stress values evaluated on all samples based on the final relation between the stress and parameter $P$ for the prestressed strands deviated from the real values about $\pm 2.0 \%$ of the largest stress at maximum.

The large differences between the magnetoelastic characteristics of the prestressed bars made from different materials were determined. Moreover, it was found, the screw thread of the bar influences negatively the experiment. For obtaining of acceptably accurate results 
by the magnetoelastic method, the screw thread has to be removed from the bar surface in the neighbourhood of the cross section, where the magnetoelastic sensor would be placed. And it is unrealizable practically on an existing structure in real conditions. The sensitivity of the dimensionless parameter $P$ on the mechanical stress in the investigated prestressed bars is substantially less than these ones obtained for the prestressed strands or wires. This matter of fact is caused partly by the significantly lower design resistance of the bar materials and also by their dissimilar chemical compositions (see Table 1).

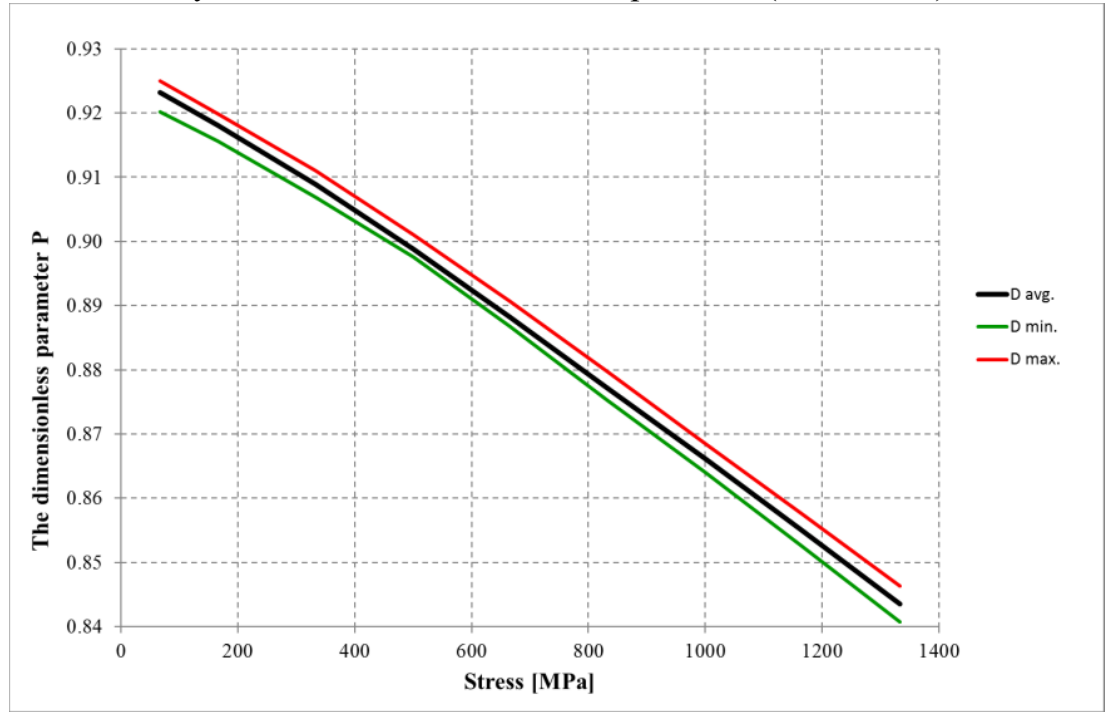

Fig. 3. The results of the statistical evaluation of the verification experiment for the patent wires D1, $\mathrm{D} 2$ and D3 and for the temperature step $+20^{\circ} \mathrm{C}$ that describes the relation between the stress and the resultant dimensionless parameter $P$ and its variation.

\section{The application in situ on the existing structure}

\subsection{The experiment description}

The usability test of the new approach described in this paper by an application in situ was carried out on a prestessed concrete structure, namely on the existing footbridge across Vltava River in Prague built in 1984.

The load bearing structure of the footbridge is built as the precast segmental construction and its static system is the stress ribbon bridge with three spans $85.5 \mathrm{~m}, 96.0 \mathrm{~m}$ and $67.5 \mathrm{~m}$. The considerable part of the footbridge deck was overflowed by two great floods that happened in August 2002 and in June 2013.

The main reason for realization of the experiment on this particular footbridge were doubts about the real actual value of the prestress in the prestressed cables that was influenced partly by their corrosion and also by partial deterioration of the construction conditions of the footbridge load bearing structure caused by two above mentioned great floods.

The basic information about the used substantial structural elements, about the load bearing structure geometry and about the installation procedure was obtained from the archived footbridge design documentation. 
There are used two types of steel cables on the footbridge structure. The cables of type A are the carrying ones of the stress ribbon bridge and the cables of type B are the prestressed ones preventing the dilatation of transversal gaps between the segments.

One magnetoelastic sensor designed for the assembly on the existing structure was installed on the prestressed cable of type B composited from six strands. The position for the sensor installation on the cable was selected not to be significantly affected by corrosion in this place. The cable was studied as the whole because of there was not space between the strands wide enough for the sensor installation on the individual strands.

At first, the hollow (see Fig. 4) was created carefully around the selected cable in which the sensor could be assembled. Secondly, the sensor body was installed on the cable. Thirdly, the secondary coil was reeled on the sensor body by the help of the specially made coiler rotating with the sensor body (see Fig. 4). Fourthly, the primary coil was made in the same way as the secondary one (see Fig. 4). Next, the system of Hall sensors was placed inside the cable in small free hallow between strands and then its position was specified precisely relative to the both coils. At the end, the thorough sensor shielding was installed (see Fig. 4).
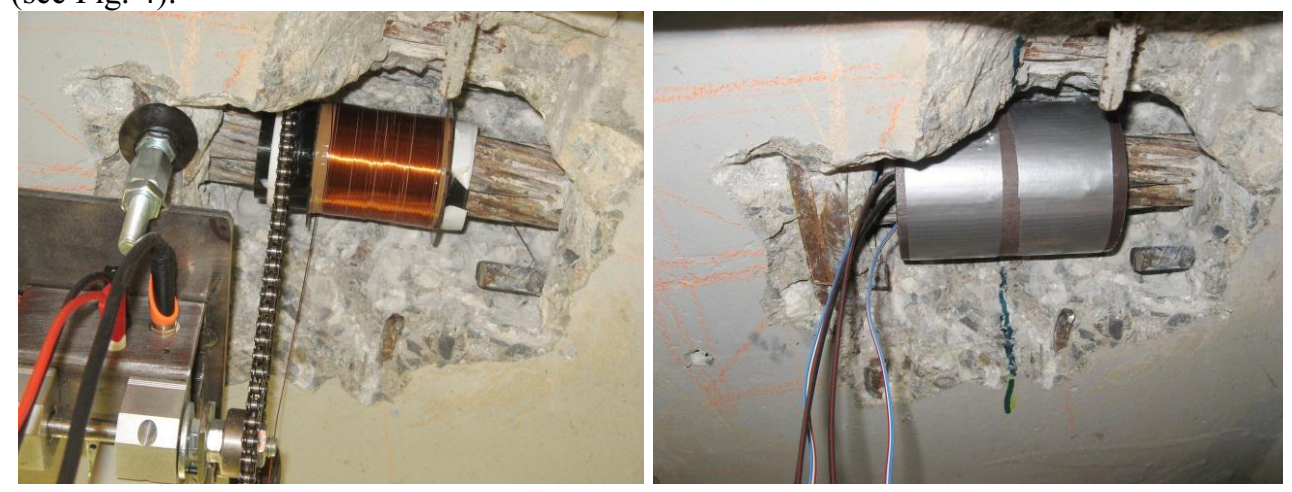

Fig. 4. The view on the created hollow, the studied cable, the coiler, the partially reeled primary coil on the black sensor body (on left) and on the completed magnetoelastic sensor (on right).

\subsection{Results of the experiment in situ}

The hysteresis loop was obtained as the primary results of the experiment. Next, the dimensionless parameter $P$ was obtained from the measured loop and then the resulting value $825 \mathrm{MPa}$ of the normal stress in the investigated cable was obtained from the evaluation curve similar to the one drawn in Fig. 3.

The assessment of acceptability of the evaluated normal stress in the cable was carried out based on comparison with expected stress in the prestressed cable that was specified from the available information about the footbridge structure. The analysis of the static calculation of the footbridge showed that the normal stress in the cables of type B could be expected in the interval from $680 \mathrm{MPa}$ to $1000 \mathrm{MPa}$. The wide range of the interval is related to the prestress losses due to successive prestressing of the subtle footbridge structure by fourteen cables of type B. A record about the real procedure of the successive prestressing of the prestressed cables during the footbridge construction enabling a more accurate assessment of the stress in the investigated cable has not been found unfortunately.

The experimentally specified resulting value of the normal stress in the investigated cable is practically located in the middle of the expected interval taken from the static calculation. It means that the experimental resulting value could be characterized as credible. 


\section{The chemical and microscopic analysis}

The chemical analysis of the selected chemical elements for the mass fraction was carried out on the same test samples described above in Chapter 3.1. The results are summarized in the Table 1.

The microscopic analysis was performed on the same test samples too. The steel uniform martensitic and tempering structure was determined for all of them except the prestressed bar T1. The strongly inhomogeneous structure was found in its cross section.

Table 1. The summarization of the results obtained from the chemical analysis of the mass fraction of the chemical elements in the test samples.

\begin{tabular}{|c|c|c|c|c|c|c|c|c|c|c|}
\hline $\begin{array}{c}\text { Test } \\
\text { sample }\end{array}$ & $\begin{array}{c}\mathrm{C} \\
{[\%]}\end{array}$ & $\begin{array}{c}\mathrm{Mn} \\
{[\%]}\end{array}$ & $\begin{array}{c}\mathrm{Si} \\
{[\%]}\end{array}$ & $\begin{array}{c}\mathrm{P} \\
{[\%]}\end{array}$ & $\begin{array}{c}\mathrm{S} \\
{[\%]}\end{array}$ & $\begin{array}{c}\mathrm{Ni} \\
{[\%]}\end{array}$ & $\begin{array}{c}\mathrm{Cr} \\
{[\%]}\end{array}$ & $\begin{array}{c}\mathrm{Co} \\
{[\%]}\end{array}$ & $\begin{array}{c}\mathrm{N} \\
{[\%]}\end{array}$ & $\begin{array}{c}\mathrm{Ba} \\
{[\%]}\end{array}$ \\
\hline $\mathrm{D} 1$ & 0.76 & 0.45 & 0.21 & 0.011 & 0.023 & 0.023 & 0.067 & 0.007 & 0.005 & $<0.01$ \\
\hline $\mathrm{D} 2$ & 0.81 & 0.70 & 0.16 & 0.022 & 0.018 & 0.030 & 0.092 & 0.005 & 0.006 & $<0.01$ \\
\hline $\mathrm{D} 3$ & 0.77 & 0.44 & 0.19 & 0.013 & 0.025 & 0.022 & 0.069 & 0.006 & 0.005 & $<0.01$ \\
\hline T3 & 0.10 & 0.92 & 0.39 & 0.014 & 0.022 & 0.170 & 0.237 & 0.013 & 0.008 & $<0.01$ \\
\hline T2 & 0.10 & 1.44 & 0.31 & 0.008 & 0.009 & 0.071 & 0.105 & 0.009 & 0.012 & $<0.01$ \\
\hline T1 & 0.21 & 1.42 & 0.53 & 0.009 & 0.008 & 0.108 & 0.125 & 0.012 & 0.011 & $<0.01$ \\
\hline L1 & 0.84 & 0.76 & 0.27 & 0.016 & 0.007 & 0.057 & 0.223 & 0.006 & 0.005 & $<0.01$ \\
\hline L2 & 0.83 & 0.83 & 0.22 & 0.011 & 0.008 & 0.035 & 0.278 & 0.006 & 0.005 & $<0.01$ \\
\hline
\end{tabular}

\section{Conclusions}

The brief principle and the basic validation results of the new approach to the experimental estimation of tensile forces in prestressed structural elements of engineering structures by the method based on the magnetoelastic principle are described in the paper. The new approach was designed and developed especially for application on existing prestressed concrete structures.

Based on the performed chemical analysis, it could be stated that the chemical elements modifying likely the magnetoelastic behaviour of the studied prestressed elements besides iron $(\mathrm{Fe})$ are also nickel $(\mathrm{Ni})$ and chromium $(\mathrm{Cr})$.

All of the experiments performed in the laboratory and on existing prestressed concrete structures in situ confirmed the applicability of the method that is very suitable for an inspection of the stress in the steel prestressed elements assembled from wires or strands on the existing prestressed concrete structures on which another nondestructive method $[1,2$, 3 ] cannot be used in the most of real cases in the civil engineering praxis.

On the other hand, the approach does not seem appropriate for inspection of the prestressed bars. These ones are usually used on civil engineering structures as temporary mounting prestressed elements and another experimental method for the tensile forces observation, as are a professionally made force transducer or a strain gauge measurement for example, could be easily used in these cases.

According to the author's knowledge, the standard magnetoelastic sensors $[4,5]$, that have hitherto been used and that have been composed from the primary and secondary coils only, are positioned on an unloaded investigated element before its activation. The sensitivity of the used measuring equipment, which is usually composed from the data logger and the sensors, is specified during tensioning of the element using the information about the actual prestressed force from a tension hydraulic jack. The standard approach is only useable for the observation of the comparative change of the tension force compared with the realized initial reading which has to be carried out in the unloaded state of the element. For the mentioned knowledge, the approach is inappropriate for an objective of the 
experimental estimation of the current value of the tensile force in a prestressed structural element on an existing concrete structure.

The described new approach also provides an advantage for experiments carried out on newly built prestressed concrete structures compared to experiments performed by standard magnetoelastic sensors $[4,5,6]$.

As was found during performed experiments, the advantage of the new approach is a possibility of the significant elimination of risks of an inaccurate evaluation of the current stress in the prestressed elements monitored already in the moment of their activation. These risks are especially attached to the possible change of magnetoelastic characteristic of the standard magnetoelastic sensor $[4,5,6]$ caused by a change of sensor magnetic surroundings in the time between its calibration during the prestressed element activation and a performed stress measuring. The variability in the sensor magnetic surroundings is common during building of a civil engineering structure. It could be caused by a concreting of steel-fibre concrete or a removal of a steel massive falsework from the sensor surroundings after concrete hardening for example.

This paper has been supported by the Technology Agency of the Czech Republic project No. TA04030307.

\section{References}

1. M. Polák, T. Plachý, Determination of Forces in Roof Cables at Administrative Center Amazon Court, Procedia Engineering 48, 578-582 (2012)

2. P. Fajman, M. Polák, J. Máca, T. Plachý, The Experimental Observation of the Prestress Forces in the Structural Elements of a Tension Fabric Structure, Appl. Mech. Mater. 486, 189-194 (2014)

3. M. Polák, T. Plachý, Experimental Evaluation of Tensile Forces in Short Steel Rods, Appl. Mech. Mater. 732, 333-336 (2015)

4. M. Chandoga, P. Fabo, A. Jaroševič, Measurement of Forces in the Cable Stays of the Apollo Bridge, Proceedings of the 2nd fib Congress, Naples, Italy, 5-8 June, 674-675 (2006)

5. A. M. Sarmento, A. Lage, E. Caetano, J. Figueiras, Stress measurement and material defect detection in steel strands by magneto elastic effect. Comparison with other nondestructive measurement techniques, Proceedings of the 6th International Conference on Bridge Maintenance, Safety and Management IABMAS 2012, Stresa, Lake Maggiore, Italy, 8 - 12 July, 914-921 (2012)

6. H. J. Wichmann, A. Holst, H. Budelmann, Magnetoelastic stress measurement and material defect detection in prestressed tendons using coil sensors, Proceedings of $7 \mathrm{th}$ International Symposium on Non-Destructive Testing in Civil Engineering NDTCE'09, Nantes, France, 30 June - 3 July (2009) 